\title{
Thrombosis of the posterior scrotal vein associated with essential thrombocytemia: Report of a case
}

\author{
Andrea Solinas \\ Department of Surgery, Unit of Urology, ATS Sardegna - ASSL Carbonia, Ospedale Sirai, Carbonia, Italy.
}

\begin{abstract}
Summary Mondor's disease is a rare superficial thrombophlebitis of subcutaneous vein and usually occurs in the anterior and lateral chest. Penile Mondor's disease is a rare condition characterized by superficial thrombophlebitis of the dorsal vein of the penis. We report a rare case of atypical penile Mondor's disease involved the right posterior scrotal vein, in a patient affected by essential thrombocythemia. A 50-years old man presented with thrombosis of right posterior scrotal vein. He presented with an indurated subcutaneous and painful cord, palpable along the length of the involved vein and located parallel to the urethra in the posterior aspect of the scrotum. It was treated with lowmolecular-weight heparin and resolves without sequelae.

The scrotal vein thrombosis is a fairly rare disease.
\end{abstract}

KEY WORDS: Mondor's disease; Superficial thrombophlebitis; Tender cord; Scrotal vein thrombosis.

Submitted 3 December 2019; Accepted 16 January 2020

\section{INTRODUCTION}

Mondor's disease is a rare superficial thrombophlebitis of subcutaneous vein and was first described by Henry Mondor in the superficial veins of the chest wall in 1939 (1). In 1955 Braun-Falco defined dorsal phlebitis of the penis in the context of generalized phlebitis while the first isolated penile Mondor's disease was defined by Helm and Hodge in 1958 (2). Penile Mondor's disease is rarer, arising out of thrombophlebitis of the penile veins. It has been reported after genital trauma such as stretching and torsion of the veins and can cause endothelial necrosis and thrombosis. It typically involves the dorsal vein of the penis and presents with a cord-like indurated lesion with a beaded feel, palpable along the length of the involved vein. The causes of the disease include frequent and prolonged sexual intercourse, prolonged sexual abstinence, infections, thrombophilia, repair of inguinal or umbilical hernia (3), orchiopexy, varicocelectomy, use of intracavernous drugs, use of vacuum, Behçet's disease, body building exercises, cancer in the pelvic region, metastatic pancreas cancer and migratory phlebitis due to paraneoplastic syndromes and tendency to thrombosis (4). Thrombosis occur as a consequence of intravascular coagulation due to injury to vessel wall, stasis, and hypercoagulation known as Virchow's triad. Though penile Mondor's disease involving the dorsal vein of the penis has been reported by many authors, atypical localization involved the circumflex vein (5) or the superficial scrotal veins (3-6) are also described. We report a rare case, involved the right posterior scrotal vein, in a patient affected by essential thrombocythemia.

\section{Case report}

A 50-years old man presented with a thrombosis of right posterior scrotal vein. He had a cord-like indurated lesion, palpable along the length of the involved vein and located parallel to the urethra in the posterior aspect of the scrotum associated with a feeling of heaviness. There was no fever, hematuria, dysuria. He gave a history of essential thrombocythemia for six months treated by interferon. Examination of genito-urinary system was normal. Standard investigations (blood and urine) requested were normal, while a blood cell count revealed $800 \times 10^{3}$ platelets. Diagnosis was confirmed on Doppler ultrasonography of the scrotal superficial venous system. It was treated with low-molecular-weight heparin for eight week and subsequently received aspirin (100 mg once daily) for prophylaxis of recurrences. He resolves without sequelae in eight weeks with recanalization of the vein and remained well three years after therapy.

\section{Discussion}

Mondor's disease is a rare superficial thrombophlebitis, historically involving the thoracic venous system. However, it can occur all over the skin. In 1955, the first reported case of Mondor's disease (superficial thrombophlebitis) of the penis was published (2). Since then there have been described reports of penile Mondor's disease in the literature. Most studies suggest neoplasm, sexual trauma, sickle cell anemia, excessive sexual activity or abstinence as the most frequent etiology and diabetes as predisposing factor due to its frequent pelvic problems leading to a potential venous inflammatory trigger. The application of immunohistochemical markers revealed that almost all Mondor's diseases appeared to be thrombophlebitis of the superficial vein. The progression of phlebitis includes some pathophysiological steps in developing Mondor's disease. At the initial stages, thrombotic events occur in the affected veins. As a result the lumen often becomes occluded with fibrin and inflammatory cells (7). Subsequently, the connective tissue gathering in the vessel forms a hard, cord-like 
induration. Thereafter, recanalization proceeds for several weeks until establishment. Mondor's disease can occur without a clearly determined aetiology but In our case, thrombocytemia was identified as a main risk factor. Diagnosis of Mondor's disease is usually made on clinical findings, thorough taking of the medical history and a correct physical examination are essential for diagnosing. The thrombosed superficial veins should first be detected with Doppler ultrasonography. Doppler ultrasonography can be also used in follow-up, showing the recanalization of the endoluminal thrombosis vein (8-9). The standard treatment strategy for Mondor's disease has not yet been established because of the paucity of clear evidence. Most cases resolve within 4 to 6 week with re-permeabilization. Currently treatment is palliative for most patients with anti-inflammatory drugs and local heparin containing creams but in our patient with thrombophilia we preferred anticoagulation with low-molecularweight heparin and subsequently with aspirin to prevent additional thrombosis. However, antibiotic therapy should be administered when cellulitis is suspected and vein stripping may be necessary for severe, persistent cases. In secondary Mondor's disease, treating the underlying disease is of the highest priority because Mondor's disease itself is not life-threatening, while the underlying diseases may lead to a lethal outcome.

\section{Conclusions}

Mondor's disease is a rare self-limiting benign process with acute presentation characterized by a cord-like induration in several parts of the body. Although its physiopathology is not exactly known, transection of the vessel during surgery or any type of trauma such as external compression may trigger its possible development. The patient usually feel the superficial vein like a hard cord and present with complaint of pain around this hardness. Diagnosis is usually easy with medical history and physical examination. Color Doppler ultrasound examination is important for differential diagnosis. The relationship between Mondor's disease and other sites of superficial thrombophlebitis remains unclear, but such lesions are considered an initial manifestation of generalized throm- bophlebitis. Some cases of Mondor disease may occur secondary to an underlying disease, such us vasculitis, a hypercoagulative state, or malignancy. The prognosis of secondary Mondor's disease therefore depends on the prognosis of the underlying disease. Physicians should correctly identify Mondor's disease, evaluate the possible presence of an underlying disease and avoid performing unnecessary invasive tests or treatment.

\section{ACKNOWLEDGMENTS}

The article is dedicated to my son Lorenzo.

\section{REFERENCES}

1. Nazir SS, Khan M. Thrombosis of the dorsal vein of the penis (Mondor's Disease): A case report and review of the literature. Indian J Urol. 2010; 26:431-3.

2. Helm JD Jr, Hodge IG. Thrombophlebitis of dorsal vein of the penis: report of a case treated by phenylbutazone (Butazolidin). J Urol. 1958; 79:306-7.

3. Mendez Rubio S, Menéndez Sánchez P, et al. Idiopathic thrombosis of the superficial scrotal veins (Mondor's disease) during the postoperative period of an umbilical herniorraphy. Arch Esp Urol. 2012; 65:903-7.

4. Öztürk H, Penile Mondor's disease. Basic Clin Androl 2014; 3:24:5.

5. Arora R, Sonthalia S, Gera T, Sarkar R. Atypical penile Mondor's disease-involvement of the circumflex vein. Int J STD AIDS. 2015; 26:360-3.

6. Fujii Y, Arisawa C, Horiuchi S, et al. Thrombosis of the posterior scrotal vein: report of two cases. Hinyokika Kiyo. 1992; 38:1417-19.

7. Amano M, Shimizu T. Mondor's disease: a review of the literature. Intern Med. 2018; 57:2607-12.

8. Ouattara A, Karim Parè A, Kaborè AF, et al. Subcutaneous dorsal penile vein thrombosis or penile Mondor's disease: a case report and literature review. Case Rep Urol. 2019; 2019:1297048.

9. Dicuio M, Pomara G, Ales V, et al. Doppler ultrasonography in a young patient penile Mondor's disease. Arch Ital Urol Androl. 2005; 77:58-9.

\section{Correspondence}

Andrea Solinas, MD (Corresponding Author)

sol.andrea@tiscali.it

S.C. Urologia, ATS Sardegna -ASSL Carbonia, Ospedale Sirai

Via Ospedale, 09013 Carbonia (Italy) 